\title{
Radial ray hypoplasia-choanal atresia syndrome
}

INSERM

\section{Source}

INSERM. (1999). Orphanet: an online rare disease and orphan drug data base. Radial ray hypoplasia-choanal atresia syndrome. ORPHA:3026

Radial ray hypoplasia - choanal atresia is an extremely rare syndrome characterized by radial ray hypoplasia, choanal atresia and convergent strabismus. 